\title{
By regulating miR-182-5p/BCL10/CYCS, sufentanil reduces the apoptosis of umbilical cord mesenchymal stem cells caused by ropivacaine
}

\author{
Lisha Li ${ }^{1,2,3}$, Yan Sun ${ }^{1,2,3}$, Na Zhang ${ }^{1,2,3}$, Xuemin Qiu ${ }^{1,2,3}$, Ling Wang ${ }^{1,2,3, *}$, Qingyan Luo ${ }^{1, *}$ \\ ${ }^{1}$ Obstetrics and Gynecology Hospital of Fudan University, Shanghai, China; \\ ${ }^{2}$ The Academy of Integrative Medicine of Fudan University, Shanghai, China; \\ ${ }^{3}$ Shanghai Key Laboratory of Female Reproductive Endocrine-related Diseases, Shanghai, China.
}

\begin{abstract}
Summary Sufentanil is a type of opioid analgesic and is usually used to facilitate painless labor in combination with the local anesthetic ropivacaine. One aim of the current study was to investigate the effects of sufentanil and ropivacaine on umbilical cord mesenchymal stem cells (UCMSCs). A second aim of this study was to determine whether sufentanil attenuated the cytotoxicity of ropivacaine in vitro. UCMSCs were divided into 3 groups: one was treated with ropivacaine at a concentration of $50,100,200$, or $400 \mu \mathrm{g} / \mathrm{mL}$, another was treated with sufentanil at a concentration of $0.5,5,50$, or $500 \mathrm{nmol} / \mathrm{L}$, and a third was treated with a combination of ropivacaine at a concentration of $200 \mu \mathrm{g} / \mathrm{mL}$ and sufentanil at a concentration of $0.5,5,50$, or $500 \mathrm{nmol} / \mathrm{L}$. Results indicated that cell proliferation decreased in cells treated with ropivacaine while it increased in cells treated with sufentanil. In addition, sufentanil limited the inhibitory effect of ropivacaine on UCMSC growth in a dose- and time-dependent manner. Combined treatment with ropivacaine at a concentration of $200 \mu \mathrm{g} / \mathrm{mL}$ and sufentanil at a concentration of $500 \mathrm{nmol} / \mathrm{L}$ decreased the proportion of dead and apoptotic UCMSCs, and fewer cells were arrested in the $\mathbf{S}$ phase compared to cells treated with ropivacaine. Sufentanil inhibited the apoptosis induced by ropivacaine by increasing miR-182-5p, which regulated the expression of mRNA of the pro-apoptotic genes B-cell lymphoma/leukemia 10 (BCL10) and cytochrome c, somatic (CYCS). Sufentanil also increased the expression of mRNA of anti-apoptotic genes. In short, ropivacaine inhibits the cell viability and induces the apoptosis of UCMSCs in vitro while sufentanil attenuates this apoptosis by regulating miR182-5p/BCL10/CYCS.
\end{abstract}

Keywords: Sufentanil, ropivacaine, umbilical cord mesenchymal stem cells, cell cycle arrest, apoptosis, miR-182-5p, BCL10, CYCS

\section{Introduction}

The pain of childbirth can cause a series of neuroendocrine

Released online in J-STAGE as advance publication February 17, 2019.

*Address correspondence to:

Dr. Qingyan Luo, Hospital and Institute of Obstetrics and Gynecology, Fudan University, 419 Fangxie Road, Shanghai 200011, China.

E-mail: wsqlqy@126.com

Dr. Ling Wang, Hospital and Institute of Obstetrics and Gynecology, Fudan University, 419 Fangxie Road, Shanghai 200011, China.

E-mail: Dr.wangling@fudan.edu.cn responses, reduce the placenta blood flow, and cause vasoconstriction and acidosis; these adverse events negatively affect the fetus and mother. Effective labor analgesia can reduce the incidence of these adverse events, shorten the birth process, and reduce energy consumption and labor pains $(1,2)$. Neuraxial anesthesia is considered to be the gold standard for the control of labor pain. Epidural analgesia is considered to be the most effective method of labor analgesia. It is ideal as analgesia and can also be used as anesthesia for a caesarean section in the event that a vaginal delivery is not possible; it also beneficial to the fetus because it increases blood flow through the placenta and it allows a pregnant woman to remain awake so that she can 
cooperate with the physician $(3,4)$.

Synergy between opioids and local anesthetics further increases the quality of analgesia and decreases the dose requirement of both local anesthetics and opioids (5). Ropivacaine, as a new local anesthetic, has little cardiac toxicity and no obvious effect on the blood flow of the uterus and placenta. It has a direct role on the nerve roots or spinal cord, it does not cause fetal respiratory inhibition, and it is an effective analgesic (6). Sufentanil is a strong opioid analgesic that is quick-acting, it has a short half-life of elimination, and it has little effect on hemodynamics (7). A combination of ropivacaine and sufentanil is a highly effective form of labor analgesia that can effectively relieve pain, shorten the course of labor, reduce postpartum hemorrhage, and reduce the need to perform a cesarean section (8). However, whether these drugs have an adverse effect on newborn's prognosis is not known.

On the one hand, anesthesia can inhibit the stress reaction caused by surgery and pain, but on the other hand it can also directly or indirectly affect cell function. In clinical settings, studies have found that drugs have no adverse effect on physical and mental development $(9,10)$, but other studies with different concentrations of anesthetics have reported that residual levels of those drugs are detectable in the cord blood and breast milk (11).

Umbilical cord mesenchymal stem cells (UCMSCs) are multi-functional stem cells located in the umbilical cord tissue of the newborn. These cells can differentiate into a variety of tissue cells and they offer promise for their clinical use. In the process of pregnancy and childbirth, the umbilical cord plays an important role in metabolism; substances in the umbilical cord bloodstream play an important role in the function of the umbilical cord $(12,13)$. The effects of sufentanil and ropivacaine on human UCMSCs alone or in combination is still not known.

The current study investigated the cytotoxicity of sufentanil and ropivacaine in UCMSCs alone or in combination, and this study also examined the functions of those drugs. Those findings were analyzed to determine if a combination of sufentanil and ropivacaine could serve as a potential form of anesthesia that reduces cell damage.

\section{Materials and Methods}

\subsection{Cell culture}

Human UCMSCs were purchased from the Shanghai Branch of the Chinese Academy of Science and cultured in Dulbecco's modified Eagle's medium (DMEM) containing $10 \%$ fetal bovine serum (FBS), $100 \mathrm{U} / \mathrm{mL}$ penicillin, and $100 \mathrm{mg} / \mathrm{mL}$ streptomycin at $37^{\circ} \mathrm{C}$ in a $5 \%$ $\mathrm{CO}_{2}$ atmosphere.

\subsection{Drug treatments}

UCMSCs in the logarithmic growth phase were randomly divided into 4 groups: a blank control group (C), a group of cells treated with ropivacaine (Ro), a group of cells treated with sufentanil (Su), and a group of cells treated with a combination of ropivacaine and sufentanil $(\mathrm{Ro}+\mathrm{Su})$. Cells treated with ropivacaine were divided into 4 groups (Ro 50, Ro 100, Ro 200, and Ro 400) depending on the concentration of ropivacaine $(50,100,200$, and $400 \mu \mathrm{g} / \mathrm{mL})$. Cells treated with sufentanil were divided into 4 groups ( $\mathrm{Su} \mathrm{0.5,} \mathrm{Su} \mathrm{5,}$ $\mathrm{Su} 50$, and Su 500) depending on the concentration of sufentanil $(0.5,5,50$, and $500 \mathrm{nmol} / \mathrm{L})$. Cells treated with a combination of ropivacaine and sufentanil were also divided into 4 groups treated with ropivacaine at a concentration of $200 \mu \mathrm{g} / \mathrm{mL}$ along with different concentrations of sufentanil $(0.5,5,50$, and $500 \mathrm{nmol} / \mathrm{L})$ (Ro $200+$ Su 0.5, Ro $200+$ Su 5, Ro $200+$ Su 50, and Ro $200+$ Su 500).

\subsection{Cell transfection}

UCMSCs were seeded in 6-well plates overnight, treated with different drugs, and then transiently transfected with miR-182-5p inhibitors or mimics with Lipofectamine 2000 (Invitrogen, CA, USA). miR-182$5 p$ mimics (mimics), an miR-182-5p-negative control (miR-NC), an anti-miR-182-5p inhibitor (anti-miR), and a negative control (anti-NC) were synthesized by GenePharma (Shanghai, China). The effects of gene silencing or overexpression were measured using realtime PCR $48 \mathrm{~h}$ after transfection.

\subsection{Real-time cell analyzer (RTCA) system}

The xCELLigence RTCA DP System (ACEA Biosciences, San Diego, California, USA) allows label-free and real-time monitoring of cellular processes, such as cell proliferation, cytotoxicity, adhesion, viability, invasion, and migration using electronic cell sensor array technology. The electrode impedance, displayed as cell index (CI) values, provides quantitative information about the biological status of cells, including their number, viability, and morphology. In brief, $50 \mu \mathrm{L}$ of cell culture medium at room temperature was added into each well of the E-plate 16 of the xCELLigence RTCA DP System. The E-plate 16 was then connected and checked in the cell culture incubator for proper electrical contacts, and the background impedance was measured for $24 \mathrm{~h}$. The UCMSCs were resuspended in cell culture medium and adjusted to 5,000 cells/well. The cell suspension (100 $\mu \mathrm{L}$ ) was added to $50 \mu \mathrm{L}$ of medium containing wells on the E-plate 16 in order to determine the optimum cell concentration. After incubation at room temperature for $30 \mathrm{~min}$, the E-plate 16 was placed into the cell culture 
incubator. Cell adhesion, growth, and proliferation were monitored every $\mathrm{h}$ for a period of up to $24 \mathrm{~h}$ via the incorporated sensor electrode arrays of the E-Plate 16. After $24 \mathrm{~h}$, different concentrations of sufentanil or ropivacaine were added to $200 \mu \mathrm{L}$ of cell culture medium, and live cells were monitored every $15 \mathrm{~min}$ for a period of up to $96 \mathrm{~h}$. The electrical impedance was measured using the RTCA-integrated software of the xCELLigence System as a dimensionless parameter termed CI.

\subsection{RNA extraction and real-time RT-PCR}

For PCR analysis, total RNA was isolated with an RNA extraction Kit (Axygen, CA, USA) according to the manufacturer's protocol. RNA purity and concentrations were measured with a NanoDrop 2000c (Thermo, Fisher, MA, USA). RNA (1 $\mu \mathrm{g})$ was transcribed into cDNA using reverse transcriptase (Promega, Madison, USA). Normalization controls for mRNA and miRNA were GAPDH and U6 RNA, respectively. Threshold cycle $(\mathrm{Ct})$ values were calculated using the software supplied with the Applied Biosystems 7900 Real-time PCR system. Primer sequences and gene abbreviations are listed in Table 1.

\subsection{Measurement of apoptosis}

After treatment with ropivacaine at a concentration of 200 $\mu \mathrm{g} / \mathrm{mL}$, sufentanil at a concentration of $500 \mathrm{nmol} / \mathrm{L}$, or a combination of the two, apoptotic cells were identified using the Annexin V-FITC Apoptosis Detection Kit according to the manufacturer's instructions. UCMSCs were treated with different drugs for $48 \mathrm{~h}$, about $1 \times$ $10^{6}$ cells from each group were stained with Annexin V-FITC for $30 \mathrm{~min}$ at $4 \mathrm{oC}$ in the dark, cells were stained with propidium iodide for $10 \mathrm{~min}$, and then cells were analyzed with a CyAN ADP flow cytometer (Beckman Coulter, Brea, CA, USA). Each sample was examined twice.

\subsection{Cell cycle analysis}

Briefly, UCMSCs were treated with ropivacaine at a concentration of $200 \mu \mathrm{g} / \mathrm{mL}$, sufentanil at a concentration of $500 \mathrm{nmol} / \mathrm{L}$, or a combination of the two for $48 \mathrm{~h}$, washed twice with ice-cold PBS, treated with trypsin, and finally fixed in cold $70 \%$ ethanol for 30 min at $4{ }^{\circ} \mathrm{C}$. The following procedures were carried out in accordance with the manufacturer's instructions for the Cell Cycle and Apoptosis Analysis Kit. Flow cytometry was used to analyze the treated cells.

\subsection{Real-time PCR arrays}

Each PCR array was a 384-well plate containing genespecific optimized real-time PCR primer sets for 84 genes related to the induction and inhibition of apoptosis. The PCR array includes primer pairs to quantify RNA18s as a positive control (an invariant highly expressed endogenous control) and genomic DNA as a negative control to detect the presence of genomic DNA contamination. Gene expression profiles were analyzed according to the manufacture's protocol (Wcgene Biotech, Shanghai, China), and data was analyzed using software from Wcgene Biotech. Genes with fold-changes more than or less than 2.0 were considered to have biological significance.

\subsection{Statistical analysis}

Each experiment was performed in sextuplicate and repeated at least three times to ensure replicability. Results were analyzed via one-way analysis of variance (ANOVA) using the software GraphPad Prism (version 5.0, GraphPad Software, La Jolla, CA, USA), followed by Dunnett's $t$-test. $P<0.05$ was considered to be significant.

\section{Results}

3.1. Sufentanil limited the inhibitory effect of ropivacaine on UCMSC growth in a time- and concentrationdependent manner

This study examined the effects of ropivacaine and sufentanil on the growth of UCMSCs. UCMSCs were treated with different concentrations of ropivacaine,

Table 1. The primers used in the study

\begin{tabular}{lll}
\hline Gene & Forward Primer & Reverse Primer \\
\hline$m i R-182-5 p$ & TGCGGTTTGGCAATGGTAGAAC & CCAGTGCAGGGTCCGAGGT \\
$B C L 10$ & GGAGTGTGAGCCACCTAAG & CTGGGCGATAGAGCAAGAC \\
CYCS & AAGGCATCACCTGGGGAGAG & ACAGAAACATTCCATCAGCCAT \\
$U 6$ & CTCGCTTCGGCAGCACA & AACGCTTCACGAATTGCGT \\
GAPDH & AATCCCATCACCATCTTC & AGGCTGTTGTCATACTTC \\
\hline
\end{tabular}

Abbreviations: APAF1: apoptotic peptidase-activating factor 1; BAK1: Bcl-2 homologous antagonist/killer; $B C L 2 L 2$ : BCL2-like protein 2; BCL10: B-cell CLL/lymphoma 10; BID: BH3-interacting domain death agonist; BIRC3: Baculoviral IAP repeat-containing protein 3; BNIP3: BCL2interacting protein 3; CFLAR: CASP8 and FADD-like apoptosis regulator; CYCS: cytochrome c, somatic; FADD: Fas-associated protein with a novel death domain; IGF1R: insulin-like growth factor 1 receptor; NOD1: nucleotide-binding oligomerization domain-containing protein 1; TNFRSF9: tumor necrosis factor receptor superfamily member 9; TNFRSF11B: TNF receptor superfamily member $11 \mathrm{~b}$; TNFRSF21: TNF receptor superfamily member 21;TP53BP2: tumor protein $\mathrm{p} 53$-binding protein 2; TRAF3: TNF receptor-associated factor 3 
sufentanil, or a combination of the two, and then the xCELLigence RTCA DP System was used to monitor cell proliferation in real time. Ropivacaine was found to inhibit the proliferation of UCMSCs (Figure 1A) while sufentanil promoted the proliferation of UCMSCs (Figure 1B). Sufentanil markedly limited the inhibitory effect of ropivacaine on UCMSC growth in a time- and concentration-dependent manner in the cells treated

A

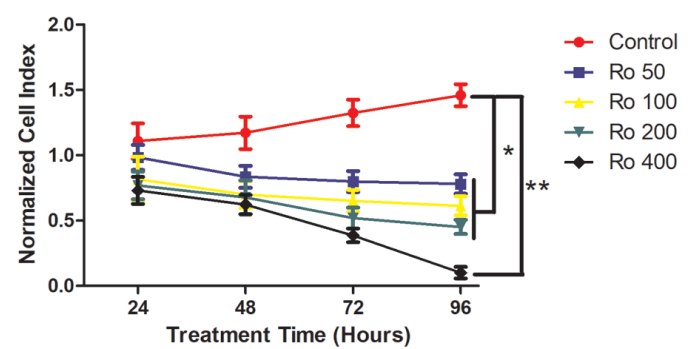

with a combination of ropivacaine and sufentanil (Figure 1C).

\subsection{Sufentanil inhibited the apoptosis of UCMSCS caused by ropivacaine and it influenced the cell cycle}

To determine whether ropivacaine or sufentanil would induce the proliferation of UCMSCs, whether the drugs

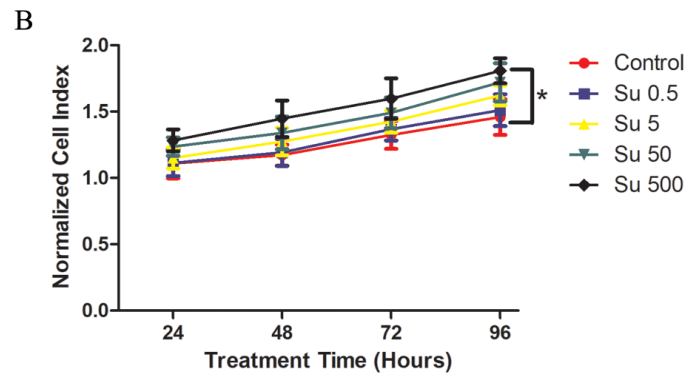

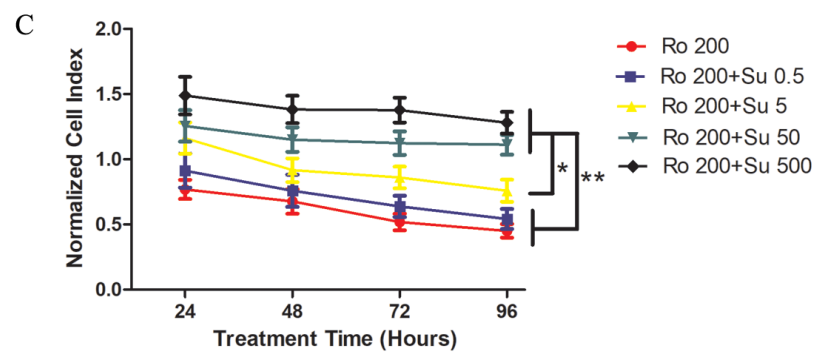

Figure 1. Effect of different concentrations of ropivacaine or sufentanil or a combination of the two on cell proliferation of cultured UCMSCs after treatment for $\mathbf{2 4}, \mathbf{4 8}, \mathbf{7 2}$, or $96 \mathbf{h}$. (A) Ropivacaine inhibited the proliferation of UCMSCs in a time- and concentration-dependent manner. (B) Sufentanil promoted the proliferation of UCMSCs in a time- and concentrationdependent manner. (C) Sufentanil limited the inhibitory effect of ropivacaine on UCMSC growth in a time- and concentrationdependent manner. ${ }^{*} p<0.05$. All results are expressed as the mean $\pm \mathrm{SEM}$, and data are representative of at least three experiments.

A

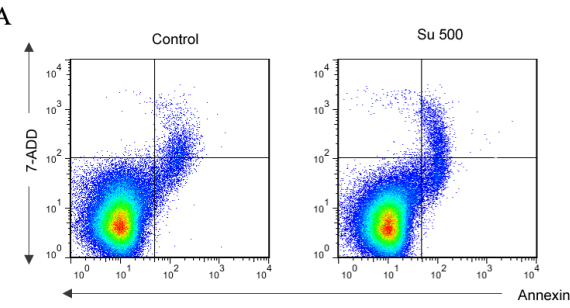

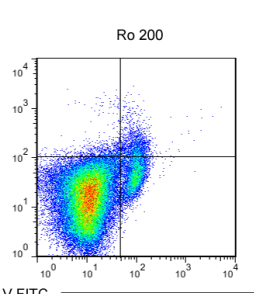

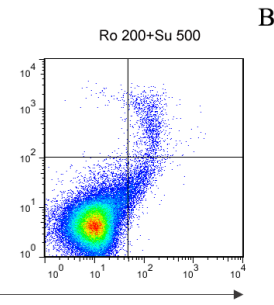

B

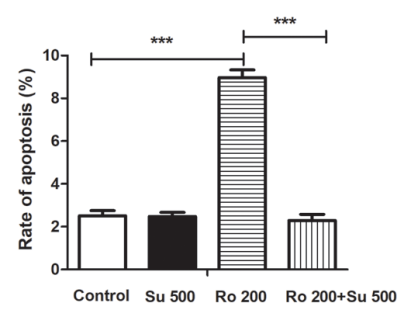

$\mathrm{C}$

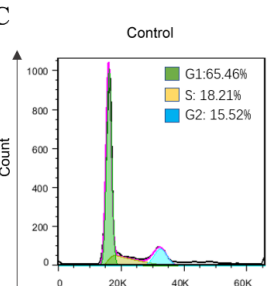

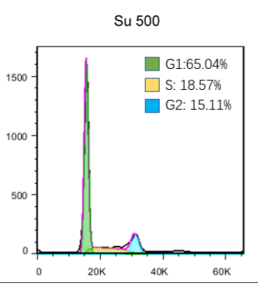

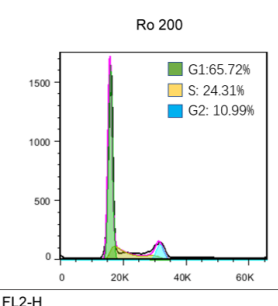

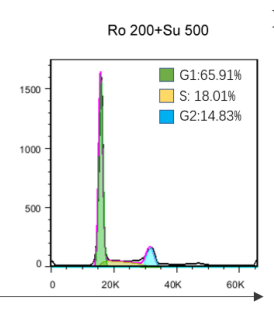

$\mathrm{D}$

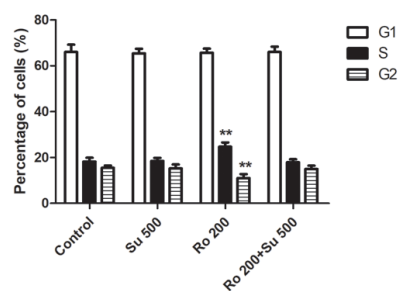

Figure 2. Effect of ropivacaine, sufentanil, or a combination of the two on cell apoptosis and the cell cycle of cultured UCMSCs after treatment for $48 \mathrm{~h}$. (A) Flow cytometry dot plots indicating the binding of Annexin V-FITC (FL-1) and PI uptake (FL-3) of UCMSCs treated with different drugs for 48 h. (B) Rates of apoptotic cells as shown in A. (C) Cell cycle analysis of UCMSCs treated with different drugs for $48 \mathrm{~h}$. The G1, S, and G2 phases are displayed in three different colors in each chart. (D) The percentage of UCMSCs in the G1, S, and G2 phases as shown in (C). $* p<0.05$. All results are expressed as the mean $\pm \mathrm{SEM}$, and data are representative of at least three experiments. 
would affect the apoptosis of those cells was determined. As shown in Figure 2A and 2B, the percentage of apoptotic cells increased in cells treated with ropivacaine at a concentration of $200 \mu \mathrm{g} / \mathrm{mL}$ while treatment with sufentanil at a concentration of $500 \mathrm{nmol} / \mathrm{L}$ did not affect apoptosis. A combination of the two drugs decreased the apoptosis of UCMSCs in comparison to cells treated with ropivacaine. Flow cytometry was used to determine whether the promotion of UCMSC proliferation by sufentanil was mediated, at least in part, by affecting cell cycle progression. As shown in Figure 2C and 2D, treatment with ropivacaine at a concentration of $200 \mu \mathrm{g} /$ $\mathrm{mL}$ significantly increased the percentage of UCMSCs in the $\mathrm{S}$ phase and decreased the percentage in the $\mathrm{G} 2$ phase in comparison to the control group and the cells treated with sufentanil at a concentration of $500 \mathrm{nmol} / \mathrm{L}$. In contrast, treatment with a combination of ropivacaine and sufentanil decreased the number of cells in the $\mathrm{S}$ phase and increased the number in the G2 phase compared to cells treated with ropivacaine. These findings indicated that ropivacaine arrested the cell cycle in the $\mathrm{S}$ phase and that sufentanil halted that arrest, suggesting that sufentanil modulates the inhibition of cell proliferation by ropivacaine by modulating the $\mathrm{S}$ phase of the cell cycle.

\subsection{Sufentanil attenuated ropivacaine cytotoxicity by regulating apoptosis-related genes}

PCR arrays were used to study the expression of 84 key genes involved in programmed cell death. These 84 genes included those that induced apoptosis, those that inhibited apoptosis, and those that coded for death domain proteins, caspases, and regulators (Figure $3 \mathrm{~A})$. Treatment with ropivacaine at a concentration of $200 \mu \mathrm{g} / \mathrm{mL}$ was found to significantly increase the expression of mRNA of most pro-apoptotic genes such as BCL10, CYCS, FADD, and the caspase family (Figure $3 \mathrm{~B})$ while treatment with sufentanil at a concentration of $500 \mathrm{nmol} / \mathrm{L}$ mainly decreased the expression of mRNA of pro-apoptotic genes and increased the expression of mRNA of anti-apoptotic genes such as BIRC3, BNIP3, and $B N I P 3 L$ (Figure 3C). Combined treatment with ropivacaine and sufentanil attenuated apoptosis caused by ropivacaine by markedly decreasing the expression of mRNA of pro-apoptotic genes such as BAK1, BCL10, $C A S P 1, C A S P 4, C Y C S$, and FADD and by increasing the expression of mRNA of anti-apoptotic genes such as BIRC3 and BNIP3 (Figure 3D).

\subsection{MiR-182-5p participated in regulating cell proliferation induced by sufentanil and ropivacaine}

Based on the apoptosis-regulating genes expressed in cells treated with a combination of ropivacaine and sufentanil, several micro-RNAs that may participate in modulating apoptosis were identified. Results indicated

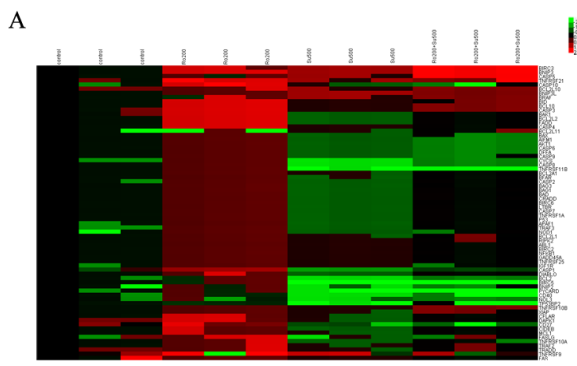

\section{B}
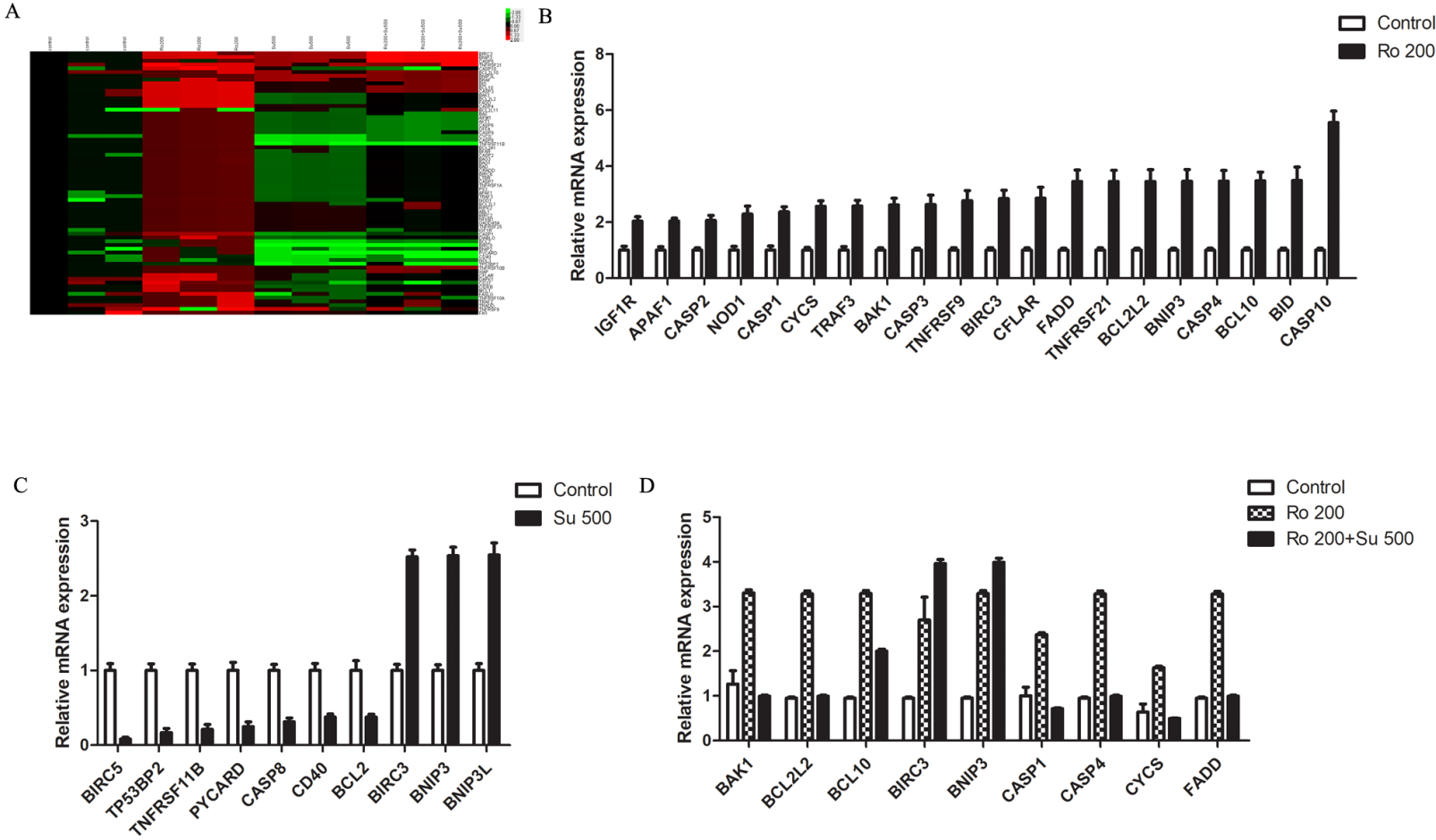

Figure 3. Sufentanil attenuated ropivacaine cytotoxicity by regulating apoptosis-related genes. (A) Eighty-four apoptosisrelated genes were analyzed using a PCR array. (B) The levels of expression of mRNA of apoptosis-related genes increased as a result of treatment with ropivacaine at a concentration of $200 \mu \mathrm{g} / \mathrm{mL}$. (C) The levels of expression of mRNA of apoptosis-related genes were modulated by sufentanil at a concentration of $500 \mathrm{nmol} / \mathrm{L}$. (D) The levels of expression of mRNA of pro-apoptotic and anti-apoptotic genes were modulated by ropivacaine and altered by a combination of ropivacaine and sufentanil. Significance is indicated in all of the bar graphs with ${ }^{*} p<0.05$. All results are expressed as the mean \pm SEM, and data are representative of at least three experiments. 

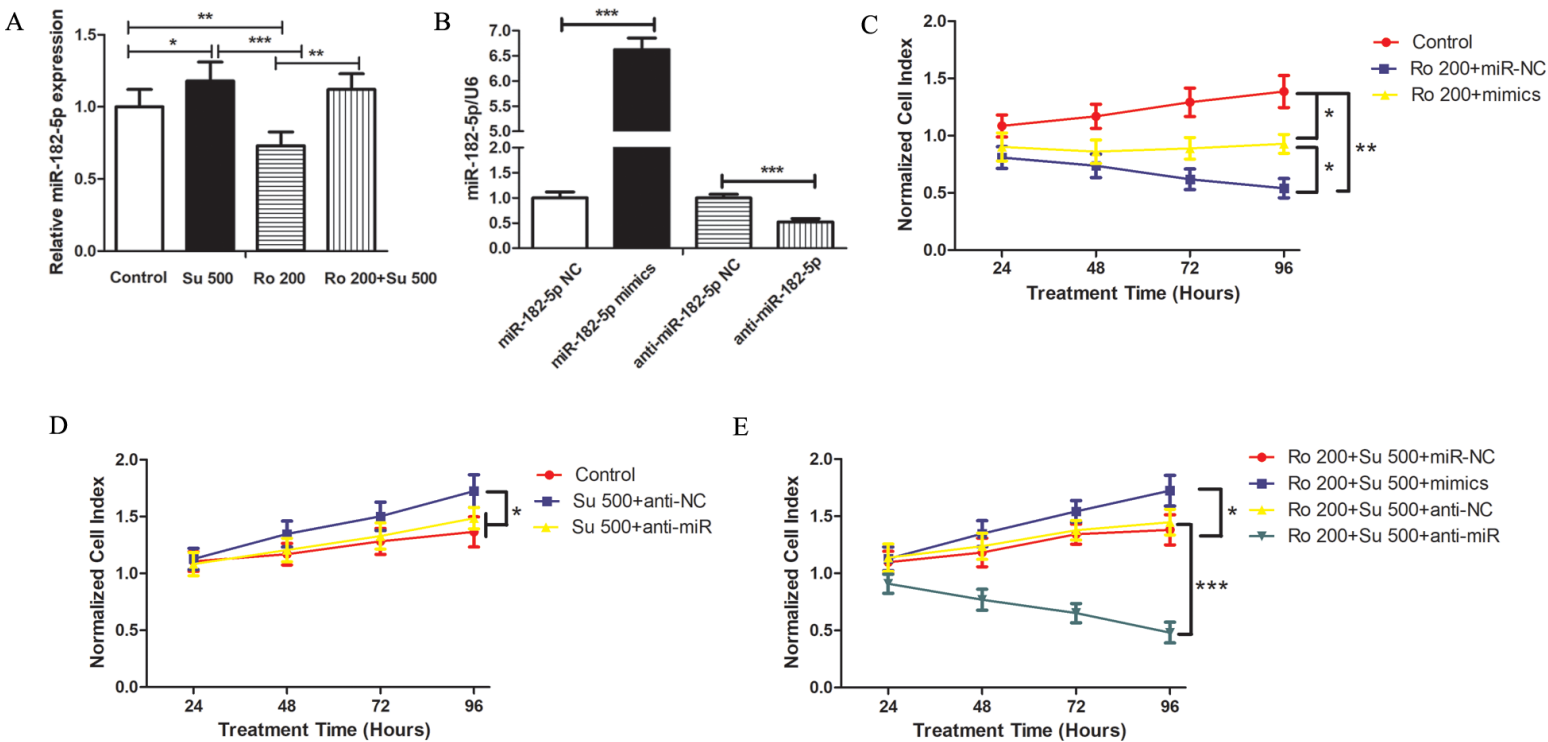

Figure 4. Sufentanil or ropivacaine modulated miR-182-5p expression to regulate cell proliferation (A) The levels of expression of miR-182-5p mRNA in each treatment group after treatment for $48 \mathrm{~h}$. (B) The levels of expression of miR-182-5p mRNA in UCMSCs $48 \mathrm{~h}$ after transfection with miR-182-5p mimics, inhibitors, or a negative control (NC). (C) MiR-182-5p mimics attenuated the inhibition of cell proliferation by ropivacaine. (D) MiR-182-5p inhibitors attenuated the promotion of cell proliferation by sufentanil. (E) MiR-182-5p mimics improved cell proliferation while inhibitors attenuated cell proliferation in cells treated with a combination of ropivacaine and sufentanil. ${ }^{*} p<0.05$. All results are expressed as the mean \pm SEM, and data are representative of at least three experiments.

that treatment with sufentanil at a concentration of 500 $\mathrm{nmol} / \mathrm{L}$ increased the level of miR-182-5p which had decreased as a result of treatment with ropivacaine at a concentration of $200 \mu \mathrm{g} / \mathrm{mL}$ (Figure $4 \mathrm{~A}$ ). miR-182$5 p$ mimics or miR-182-5p inhibitors (Figure 4B) were used to determine cell proliferation in response to treatment with different drugs. Transfection with miR182-5p mimics was found to improve cell proliferation in cells treated with ropivacaine at a concentration of $200 \mu \mathrm{g} / \mathrm{mL}$ (Figure 4C) and cells treated with a combination of ropivacaine and sufentanil (Figure 4E), while transfection with miR-182-5p inhibitors was found to attenuate cell proliferation in cells treated with sufentanil at a concentration of $500 \mathrm{nmol} / \mathrm{L}$ (Figure 4D) and cells treated with a combination of ropivacaine and sufentanil (Figure 4E).

\subsection{Sufentanil modulated miR-182-5p to inhibit apoptosis caused by ropivacaine by targeting BCL10 and $C Y C S$}

To look for the target genes of miR-182-5p in cells rendered apoptotic by drug treatment, online prediction software (TargetScan 7.0 and miRBase) was used to search for the most likely target genes. Results indicated that $B C L 2, B C L 10$, and $C Y C S$ were the likely target genes of miR-182-5p (Figure 5A). Since $B C L 2$ is a proven target of miR-182-5p, the current results verified that $B C L 2$ increased in cells treated with sufentanil at a concentration of $500 \mathrm{nmol} / \mathrm{L}$ and transfected with inhibitors (Figure 5C). Expression of $B C L 10$ and CYCS mRNA decreased in cells treated with ropivacaine at a concentration of $200 \mu \mathrm{g} / \mathrm{mL}$ (Figure 5B) and cells treated with a combination of ropivacaine and sufentanil (Figure 5D) while it increased in cells treated with sufentanil at a concentration of $500 \mathrm{nmol} /$ $\mathrm{L}$ (Figure 5C) and cells treated with a combination of ropivacaine and sufentanil (Figure 5D). miR-182-5p mimics were found to attenuate apoptosis induced by ropivacaine while miR-182-5p inhibitors increased the percentage of apoptotic cells (Figure 5E).

\section{Discussion}

The current results indicated that treatment with ropivacaine alone induced cytotoxicity in UCMSCs by inhibiting proliferation, impacting the cell cycle, and promoting apoptosis, while treatment with sufentanil had the opposite effect. A combination of ropivacaine and sufentanil attenuated that cytotoxicity by modulating apoptosis-related genes. Several studies on use of a combination of ropivacaine and sufentanil to facilitate painless labor or a cesarean section have found no abnormalities in the clinical assessment of the fetus, liver function, renal function, or routine blood results, but the possible effects of a small amount of the drugs on the newborn over the long-term remain unclear. The current results warrant re-examination of the effects of supplementing narcotics with local anesthetics for labor analgesia.

The current study involved real-time detection of cell proliferation, and results indicated that cell viability decreased markedly as the concentration of ropivacaine increased. However, treatment with 
A

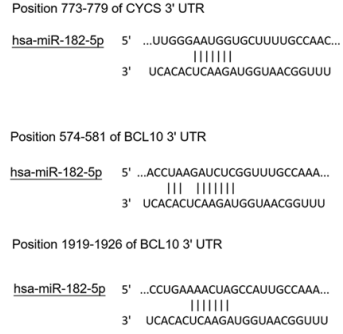

B

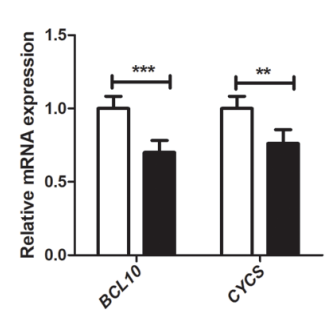

$\mathrm{C}$

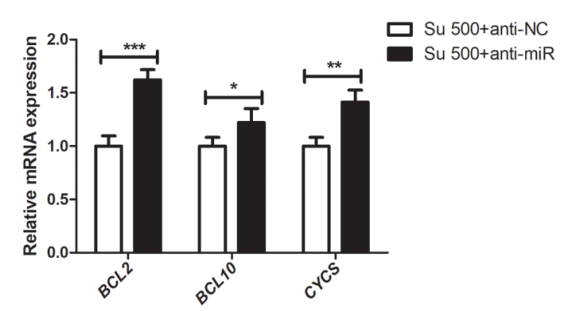

$\mathrm{D}$

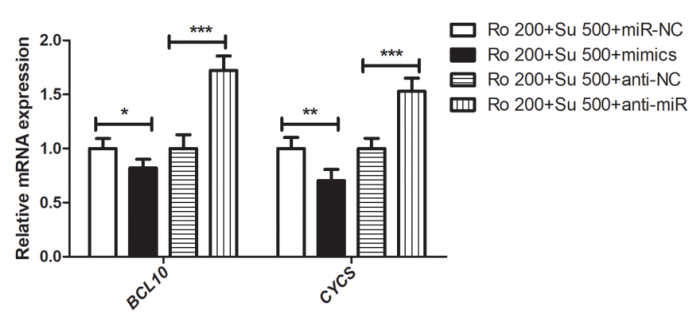

E

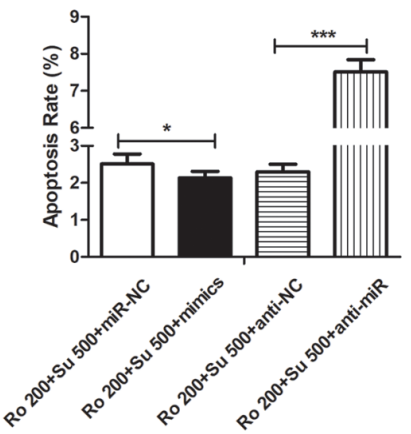

Figure 5. MiR-182-5p improved cell survival and suppressed cell apoptosis by targeting BCL10 and CYCS expression. (A) $B C L 10$ and $C Y C S$ were predicted to be target genes of miR-182-5p. (B) The levels of expression of BCL10 and CYCS mRNA decreased in cells treated with ropivacaine after transfection with miR-182-5p mimics (C) The levels of expression of $B C L 2$, $B C L 10$, and $C Y C S$ mRNA increased in cells treated with sufentanil after transfection with miR-182-5p inhibitors. (D) MiR-182-5p mimics decreased the levels of expression of BCL10 and CYCS mRNA while inhibitors increased those levels in cells treated with a combination of ropivacaine and sufentanil. (E) MiR-182-5p mimics inhibited apoptosis while inhibitors increased apoptosis in cells treated with a combination of ropivacaine and sufentanil. ${ }^{*} p<0.05$. All results are expressed as the mean $\pm \mathrm{SEM}$, and data are representative of at least three experiments.

an increasing concentration of sufentanil alone or in combination improved cell viability. In clinical studies, ropivacaine has a low toxicity when administered at a low concentration to provide a motor and sensory nerve block, but adverse reactions to ropivacaine have also been reported $(14,15)$. Many studies have indicated that sufentanil inhibits the growth of cancer cells such as human breast cancer cells or colon and pancreatic cancer cells in vitro $(16,17)$. Cord blood contains hematopoietic stem cells that can restore the human hematopoietic and immune systems. Sufentanil may improve cell viability by protecting cell or organ development when a residual amount of the drug enters the bloodstream of a fetus or baby through the umbilical cord or breastfeeding (18).

Apoptosis is an active process of intracellular programmed death that is caused by physiological and pathological changes to remove redundant and damaged cells (19). In the current study, UCMSCs were increasingly arrested in the $\mathrm{S}$ phase and displayed more apoptotic characteristics as the concentration of ropivacaine increased. In the tumor microenvironment, sufentanil attenuates cytotoxicity by inhibiting connexin 43-composed gap junction function in the treatment of colorectal cancer (20). Ropivacaine reversibly reduced the permeability of the nerve fiber membrane to sodium ions, it slowed the speed of depolarization, and it alleviated stress (21). Peroxides, DNA damage, and calcium homeostasis may induce neuron apoptosis, and mitochondrial dysfunction may activate metabolic changes related to apoptosis (22). According to the PCR array of apoptosis-related genes, ropivacaine modulated apoptosis by significantly increasing the expression of mRNA of apoptosis-related genes. This included genes that positively regulate apoptosis such as $B A K 1$, $B I D, B N I P 3$, and $C Y C S$, the death domain receptors FADD, NOD1, and TNFRSF9, the death domain proteins TNFRSF21 and TRAF3, a gene that negatively regulates apoptosis such as $B C L 10$, and the FADD-like apoptosis regulator CFLAR. Caspases can be divided into initiators such as CASP2, CASP8, CASP9, and $C A S P 10$, and effectors such as CASP3, CASP6, and CASP7. CASP1 and CASP4 are inflammatory caspases that cleave other proteins, such as the precursors of interleukins, and thus indirectly induce apoptosis (2325). Ropivacaine modulated caspases such as CASP2, $C A S P 3, C A S P 4$ and $C A S P 10$ and caspase activators such as $A P A F 1$ and $C A S P 1$ to induce apoptosis. It also increased anti-apoptotic genes such as $I G F 1 R, B I R C 3$, and $B C L 2 L 2$. Sufentanil inhibited apoptosis mainly by decreasing the expression of mRNA of pro-apoptotic genes such as TP53BP2, TNFRSF11B, and CD40 and by increasing the expression of mRNA of anti-apoptotic genes such as BIRC3 and BNIP3. In combination, sufentanil may inhibit the expression of mRNA of pro-apoptotic genes and promote the expression of 


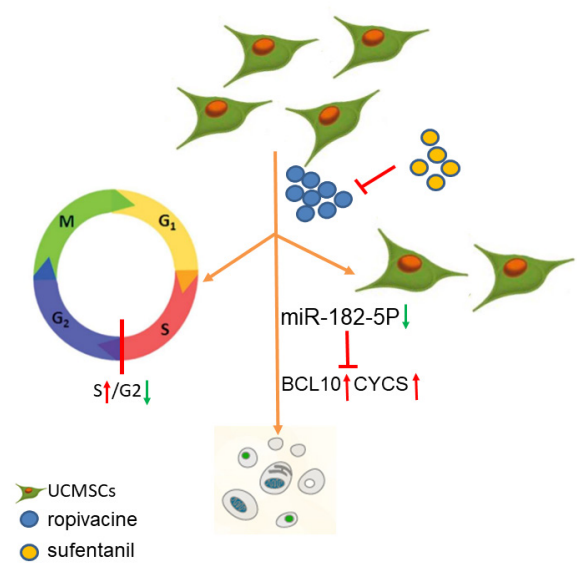

Figure 6. Schematic diagram of sufentanil reducing the apoptosis of UCMSCs caused by ropivacaine. Ropivacaine induced cytotoxicity in UCMSCs by inhibiting their proliferation, impacting the cell cycle, and promoting their apoptosis. Sufentanil improved cell viability and inhibited the apoptotic effects of ropivacaine by increasing miR-182$5 \mathrm{p}$ expression, which targeted the pro-apoptotic genes $B C L 10$ and $C Y C S$.

mRNA of anti-apoptotic genes induced by ropivacaine, even though these genes were not affected in cells treated with sufentanil. This may be due to drug interactions that lead to modulation via different signaling pathways. Sufentanil may provide protection from myocardial ischemia via the PI3K/Akt-GSK-3 $\beta$ pathway by modulating antiapoptotic proteins (26), and ropivacaine may be a potential treatment for OA by down-regulating the calcineurin/NFAT1 signaling pathway (27). However, the specific mechanism of action of these drugs need to be explored in a future study.

Studies have increasingly indicated that miRNAs play a key role in regulating gene expression during disease progression (28). MiR-182-5p has been reported to modulate cell function in many cancers. Inhibiting miR-182-5p by regulating CASP9 expression has a proapoptotic and anti-proliferative effect in human breast cancer (29). miR-182-5p promotes cell proliferation and metastasis and it inhibits apoptosis by knocking down RECK and Smad4 in bladder cancer (30), and it promotes the progression of hepatocellular carcinoma by suppressing FOXO3a expression (31). BCL2 is known to be an anti-apoptotic gene in tumors (32), and miR-182-5p regulates $B C L 2 L 12$ and $B C L 2$ expression in acute myeloid leukemia (33). However, its role as a suppressive miRNA is not clear and needs to be studied. $B C L 10$ is an apoptotic regulatory molecule that is an antigen receptor-specific regulator of NF- $\mathrm{KB}$, which is closely associated with the immune response (34). Cytochrome c $(\mathrm{Cyt} c)$, a heme-containing mitochondrial protein, has a critical function in both respiration and apoptosis. During apoptosis, cytochrome is released into the cytosol, where it subsequently participates in the process leading to caspase- 9 and caspase- 3 activation (35). The current results indicated that miR-182-5p expression was regulated in USMSCs by sufentanil or ropivacaine treatment, and that miR-182-5p improved cell survival and suppressed cell apoptosis by targeting $B C L 10$ and $C Y C S$ expression. Those drugs may play an anti-apoptotic role by regulating mitochondrial metabolism and the immune response via miR-182-5p.

Sufentanil improved cell viability and inhibited the apoptotic effects of ropivacaine by increasing miR-182$5 \mathrm{p}$ expression, which targeted the pro-apoptotic genes $B C L 10$ and $C Y C S$ in UCMSCs (Figure 6). Further studies are needed to determine the exact mechanisms for those actions. Findings from those studies could lay the foundation for combined use of those drugs in painless labor.

\section{Acknowledgements}

This work was supported by grants from the National Natural Science Foundation of China (Nos. 31571196 and 30801502 to Ling Wang), the Shanghai Program for Support of Leading Disciplines-Integrated Chinese and Western Medicine (Nos. 20180101 and 20150407), the Program to Guide Medicine ("Yixueyindao") of the Shanghai Municipal Science and Technology Commission (Nos. 18401902200 and 15401932200 to Ling Wang), the Shanghai Pujiang Program (No. 11PJ1401900 to Ling Wang), the FY2008 JSPS Postdoctoral Fellowship for Foreign Researchers (P08471 to Ling Wang), and the Fund for Young Scientists of the Shanghai Municipal Health and Family Planning Commission (No. 20184Y0218 to Lisha Li).

\section{References}

1. Onuoha OC. Epidural analgesia for labor: Continuous infusion versus programmed intermittent bolus. Anesthesiol Clin. 2017; 35:1-14.

2. Rooks JP. Labor pain management other than neuraxial: What do we know and where do we go next? Birth. 2012; 39:318-322.

3. Simmons SW, Taghizadeh N, Dennis AT, Hughes D, Cyna AM. Combined spinal-epidural versus epidural analgesia in labour. Cochrane Database Syst Rev. 2012; 10:CD003401.

4. Deshmukh VL, Ghosh SS, Yelikar KA, Gadappa SN. Effects of epidural labour analgesia in mother and foetus. J Obstet Gynaecol India. 2018; 68:111-116.

5. Kinoshita H. Novel insights into the role of anesthetics and opioids in organ or tissue protection. Curr Pharm Des. 2014; 20:5671-5672.

6. Ateser RY, Kayacan N. Intrathecal ropivacaine in cesarean delivery. Niger J Clin Pract. 2017; 20:13221327.

7. Wang Y, Tang H, Guo Q, Liu J, Liu X, Luo J, Yang W. Effects of intravenous patient-controlled sufentanil analgesia and music therapy on pain and hemodynamics after surgery for lung cancer: A randomized parallel study. J Altern Complement Med. 2015; 21:667-672.

8. Wang X, Xu S, Qin X, Li X, Feng SW, Liu Y, Wang W, Guo X, Shen R, Shen X, Wang F. Comparison 
between the use of ropivacaine alone and ropivacaine with sufentanil in epidural labor analgesia. Medicine (Baltimore). 2015; 94: e1882.

9. Erasso DM, Chaparro RE, Quiroga Del Rio CE, Karlnoski R, Camporesi EM, Saporta S. Quantitative assessment of new cell proliferation in the dentate gyrus and learning after isoflurane or propofol anesthesia in young and aged rats. Brain Res. 2015; 1441:38-46.

10. Hawkins JL. Epidural analgesia for labor and delivery. N Engl J Med. 2010; 362:1503-1510.

11. Bolat E, Bestas A, Bayar MK, Ozcan S, Erhan OL, Ustundag B. Evaluation of levobupivacaine passage to breast milk following epidural anesthesia for cesarean delivery. Int J Obstet Anesth. 2014; 23:217-221.

12. Boroujeni ME, Gardaneh M. Umbilical cord: An unlimited source of cells differentiable towards dopaminergic neurons. Neural Regen Res. 2017; 12:1186-1192.

13. Chao YH, Wu HP, Chan CK, Tsai C, Peng CT, Wu KH. Umbilical cord-derived mesenchymal stem cells for hematopoietic stem cell transplantation. J Biomed Biotechnol. 2012; 2012:759503.

14. Zheng T, Zheng CY, Zheng XC, Zhao RG, Chen YQ. Effect of parthanatos on ropivacaine-induced damage in SH-SY5Y cells. Clin Exp Pharmacol Physiol. 2017; 44:586-594.

15. Li H, Liu T, Zhu Y, Fu Q, Wu W, Deng J, Lan L, Shi S. An in situ-forming phospholipid-based phase transition gel prolongs the duration of local anesthesia for ropivacaine with minimal toxicity. Acta Biomater. 2017; 58:136-145.

16. Gong L, Qin Q, Zhou L, Ouyang W, Li Y, Wu Y, Li Y. Effects of fentanyl anesthesia and sufentanil anesthesia on regulatory $\mathrm{T}$ cells frequencies. Int J Clin Exp Pathol. 2014; 7:7708-7716.

17. Bundscherer A, Malsy M, Gebhardt K, Metterlein T, Plank C, Wiese CH, Gruber M, Graf BM. Effects of ropivacaine, bupivacaine and sufentanil in colon and pancreatic cancer cells in vitro. Pharmacol Res. 2015; 95:126-131.

18. Bauer C, Pavlakovic I, Mercier C, Maury JM, Koffel C, Roy P, Fellahi JL. Adding sufentanil to ropivacaine in continuous thoracic paravertebral block fails to improve analgesia after video-assisted thoracic surgery: A randomised controlled trial. Eur J Anaesthesiol. 2018; 35:766-773.

19. Chen QL, Gu EW, Zhang L, Cao YY, Zhu Y, Fang WP. Diabetes mellitus abrogates the cardioprotection of sufentanil against ischaemia/reperfusion injury by altering glycogen synthase kinase-3 $\beta$. Acta Anaesthesiol Scand. 2013; 57:236-242.

20. Grosse J, Warnke E, Wehland M, Pietsch J, Pohl F, Wise P, Magnusson NE, Eilles C, Grimm D. Mechanisms of apoptosis in irradiated and sunitinib-treated follicular thyroid cancer cells. Apoptosis. 2014; 19:480-490.

21. Zhang Q, Su M. Sufentanil attenuates oxaliplatin cytotoxicity via inhibiting connexin 43-composed gap junction function. Mol Med Rep. 2017; 16:943-948.
22. Gong X, Dan J, Li F, Wang L. Suppression of mitochondrial respiration with local anesthetic ropivacaine targets breast cancer cells. J Thorac Dis. 2018; 10:28042812.

23. Dai SH, Chen T, Wang YH, Zhu J, Luo P, Rao W, Yang YF, Fei Z, Jiang XF. Sirt3 protects cortical neurons against oxidative stress via regulating mitochondrial $\mathrm{Ca}^{2+}$ and mitochondrial biogenesis. Int J Mol Sci. 2014; 15:14591-14609.

24. Song GH, Huang FB, Gao JP, Liu ML, Pang WB, Li WB, Yan XY, Huo MJ, Yang X. Effects of fluoride on DNA damage and caspase-mediated apoptosis in the liver of rats. Biol Trace Elem Res. 2015; 166:173-182.

25. Wan L, Zhang D, Zhang J, Ren L. TT-1, an analog of melittin, triggers apoptosis in human thyroid cancer TT cells via regulating caspase, Bcl-2 and Bax. Oncol Lett. 2018; 15:1271-1278.

26. Wu QL, Shen T, Ma H, Wang JK. Sufentanil postconditioning protects the myocardium from ischemiareperfusion via PI3K/Akt-GSK-3 $\beta$ pathway. J Surg Res. 2012; 178:563-570.

27. Yao W, Han Q, Wang L, Niu Z. Ropivacaine relieves pain and prevents chondrocyte degradation probably through Calcineurin/NFAT1 signaling pathway in osteoarthritis rats. Eur J Pharmacol. 2018; 818:518-524.

28. Sun Z, Shi K, Yang S, Liu J, Zhou Q, Wang G, Song J, Li Z, Zhang Z, Yuan W. Effect of exosomal miRNA on cancer biology and clinical applications. Mol Cancer. 2018; 17:147.

29. Sharifi M, Moridnia A. Apoptosis-inducing and antiproliferative effect by inhibition of miR-182-5p through the regulation of CASP9 expression in human breast cancer. Cancer Gene Ther. 2017; 24:75-82.

30. Hirata H, Ueno K, Shahryari V, Tanaka Y, Tabatabai ZL, Hinoda Y, Dahiya R. Oncogenic miRNA-182-5p targets Smad4 and RECK in human bladder cancer. PLoS One. 2012; 7: e51056.

31. Cao M, You A, Zhu X, et al. miR-182-5p promotes hepatocellular carcinoma progression by repressing FOXO3a. J Hematol Oncol. 2018; 11:12

32. Delbridge AR, Strasser A. The BCL-2 protein family, BH3-mimetics and cancer therapy. Cell Death Differ. 2015; 22:1071-1080.

33. Zhang S, Zhang Q, Shi G, Yin J. MiR-182-5p regulates BCL2L12 and BCL2 expression in acute myeloid leukemia as a potential therapeutic target. Biomed Pharmacother. 2018; 97:1189-1194.

34. Gehring T, Seeholzer T, Krappmann D. BCL10 - Bridging CARDs to immune activation. Front Immunol. 2018; 9:1539.

35. Wang W, Wu J, Zhang Q, Li X, Zhu X, Wang Q, Cao $\mathrm{S}, \mathrm{Du}$ L. Mitochondria-mediated apoptosis was induced by oleuropein in H1299 cells involving activation of p38 MAP kinase. J Cell Biochem. 2018; doi: 10.1002.

(Received December 6, 2018; Revised February 7, 2019; Accepted February 10, 2019) 